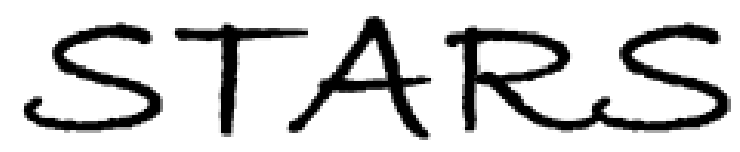

University of Central Florida

STARS

$1-1-2013$

\title{
Uniform alumina microspheres from temperature induced forming in a microfluidic T-junction
}

James Wilson

University of Central Florida

Jonathan D. Wehking

University of Central Florida

Ranganathan Kumar

University of Central Florida

Find similar works at: https://stars.library.ucf.edu/facultybib2010

University of Central Florida Libraries http://library.ucf.edu

This Article is brought to you for free and open access by the Faculty Bibliography at STARS. It has been accepted for inclusion in Faculty Bibliography 2010 s by an authorized administrator of STARS. For more information, please contact STARS@ucf.edu.

\section{Recommended Citation}

Wilson, James; Wehking, Jonathan D.; and Kumar, Ranganathan, "Uniform alumina microspheres from temperature induced forming in a microfluidic T-junction" (2013). Faculty Bibliography 2010s. 4854. https://stars.library.ucf.edu/facultybib2010/4854

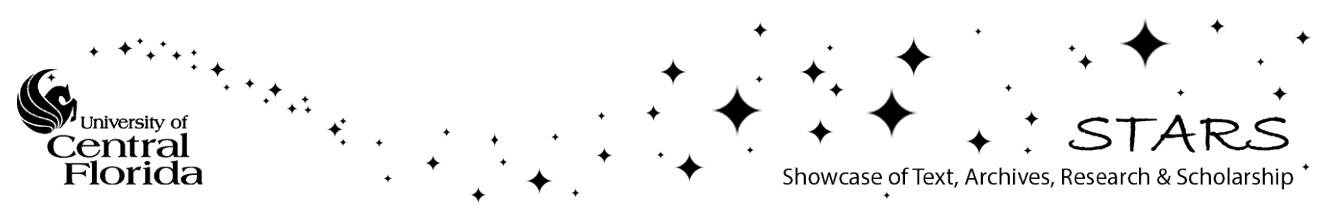




\section{Uniform alumina microspheres from temperature induced forming in a microfluidic T-junction}

Cite as: Appl. Phys. Lett. 103, 203115 (2013); https://doi.org/10.1063/1.4830358

Submitted: 24 September 2013 . Accepted: 28 October 2013 . Published Online: 15 November 2013

James Wilson, Jonathan D. Wehking, and Ranganathan Kumar
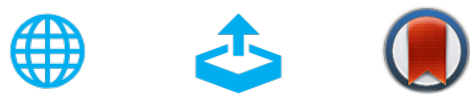

View Online

\section{ARTICLES YOU MAY BE INTERESTED IN}

Droplet deformation and manipulation in an electrified microfluidic channel

Applied Physics Letters 103, 054101 (2013); https://doi.org/10.1063/1.4817008

Flow regime transition at high capillary numbers in a microfluidic T-junction: Viscosity contrast and geometry effect

Physics of Fluids 22, 122001 (2010); https://doi.org/10.1063/1.3523483

Droplet formation and stability of flows in a microfluidic T-junction

Applied Physics Letters 94, 164107 (2009); https://doi.org/10.1063/1.3116089

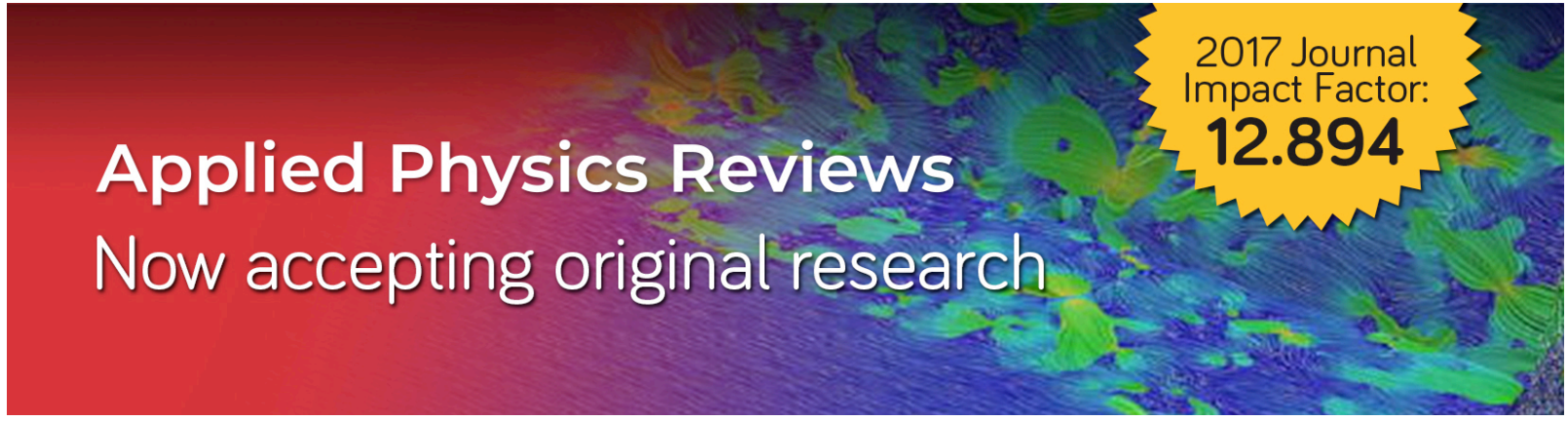




\title{
Uniform alumina microspheres from temperature induced forming in a microfluidic T-junction
}

\author{
James Wilson, Jonathan D. Wehking, and Ranganathan Kumar ${ }^{\text {a) }}$ \\ University of Central Florida, 4000 Central Florida Blvd, Orlando, Florida 32816, USA
}

(Received 24 September 2013; accepted 28 October 2013; published online 15 November 2013)

\begin{abstract}
This Letter explores a method for the manufacture of solid microspheres of $30 \mu \mathrm{m}$, from liquid droplets with nanosuspensions of $20 \mathrm{~nm}$ alumina in a microfluidic T-junction. These droplets are heated downstream where solidification takes place due to Temperature Induced Forming (TIF). TIF occurs as temperature sensitive alumina solubility leads to weakening electrostatic double-layer dispersing forces resulting in the formation of a solid structure. Manipulation of the flow characteristics and material properties of the continuous and dispersed fluids provides control of droplet production over a range of conditions and allows for a robust manufacturing facility capable of producing particles of various sizes. (c) 2013 AIP Publishing LLC. [http://dx.doi.org/10.1063/1.4830358]
\end{abstract}

For the past decade, Microfluidic devices (MFDs) have been used in several applications such as droplet generation, mixing of chemical reagents, bacterial isolation, and solid formation. Applications for these microfluidic geometries span a spectrum of industries ranging from medical and pharmaceutical to manufacturing. ${ }^{1-6}$ Research in MFDs has led to the development of parametric correlations relating flow conditions, material properties, and geometric aspects to droplet formation characteristics such as size and frequency of production for various flow regimes (squeezing, dripping, etc.). ${ }^{2,7-11}$

This Letter demonstrates that monodisperse microsolids preserving nanoscale properties can be produced in T-junction microchannels through thermal and chemical interactions at fluid/fluid interfaces. Common mechanisms for solidification have been shown to be a result of the fluid/fluid interfacial chemistry, a time delayed solidification reaction within one of the phases or by an external source such as heat or ultraviolet (UV) light within (reaction said to take place in situ) or beyond the MFD domain in some sort of reactor (reaction said to take place ex situ). ${ }^{11} \mathrm{We}$ use the combined effects of nanosuspensions of high viscosity alumina liquid droplets produced in the squeezing and dripping regimes in a $\mathrm{T}$-junction and the agglomeration characteristics of the nanoparticles inside the droplet at the interface in a thermal field to produce uniform microspheres in situ.

Work in this area has demonstrated the use of various mechanisms to induce solidification of droplets of various shapes and sizes. ${ }^{12-14}$ In many of these cases, the dispersed flow structure containing photo/thermal sensitive materials are made subject to UV light or heated environments in situ or ex situ to preserve the flow structure morphology. ${ }^{11}$

Another mechanism, Evaporation Induced Self Assembly (EISA), relies on the diffusion of the dispersing medium into the surroundings resulting in the particles assembly at the fluid/fluid interface forming a shell structure. In such miscibility studies, ${ }^{15}$ for low concentration suspensions,

\footnotetext{
${ }^{\text {a) }}$ Author to whom correspondence should be addressed. Electronic mail: Ranganathan.Kumar@ucf.edu
}

nanoparticle clusters form at the interface rendering the structure hollow.

Colloids $(<1 \mu \mathrm{m})$ with high solid loadings of alumina are commonly used to produce solid ceramic bodies by forming, drying, and sintering. The suspensions utilized and their stability characteristics depend on a balance of interparticle forces which can be affected by solid concentration, the addition of surfactants, adjustment of $\mathrm{pH}$, addition of heat, etc. ${ }^{16}$ These forces determine not only the stability of a colloid and its tendency to flocculate but also the rheological behavior of the suspension. ${ }^{16}$

For different concentrations, the gelation temperature $\left(T_{g}\right)$ varies, or the point which temperature sensitive nanoparticle suspensions begin to solidify. Just below $T_{g}$, solidification occurs but only forms weak flocs where relative increases in viscosity are small (Fig. 1). Above $T_{g}$, electrostatic double layer (EDL) forces continue to weaken and interparticle bridges form between alumina particles at higher temperatures. This leads to the formation of stronger, larger flocs, and eventually a gelled solid structure. ${ }^{16}$ The formation of interparticle networks results in an appreciable increase in viscosity for volume concentrations $>6 \%$. For concentrations $<6 \%$, viscosity increases with temperature at the gelation temperature but eventually decreases as the shearing of the fluid imposed by the viscometer destroys the bridges formed, where precipitation of the flocs from the gelled suspension occurs.

The alumina suspension was utilized to produce solid particles less than $30 \mu \mathrm{m}$ by forming a droplet upstream at a T-junction and heating a section of the microchannel downstream above $T_{g}$, thereby inducing solidification. Control over the initial droplet size produced at the microfluidic $\mathrm{T}$-junction allows for variations in particle size after solidification. The capillary number quantifies the ratio of viscous to interfacial forces and is defined by $C a=\mu_{c} U_{c} / \gamma$, where $\mu_{c}$ and $U_{c}$ are the viscosity and average velocity of the continuous liquid, and $\gamma$ is the interfacial surface tension between the continuous and dispersed liquids. The capillary number can be adjusted to produce droplets containing different volumes of dispersed fluid at varying frequencies. ${ }^{7-10,17}$ The flow rate 


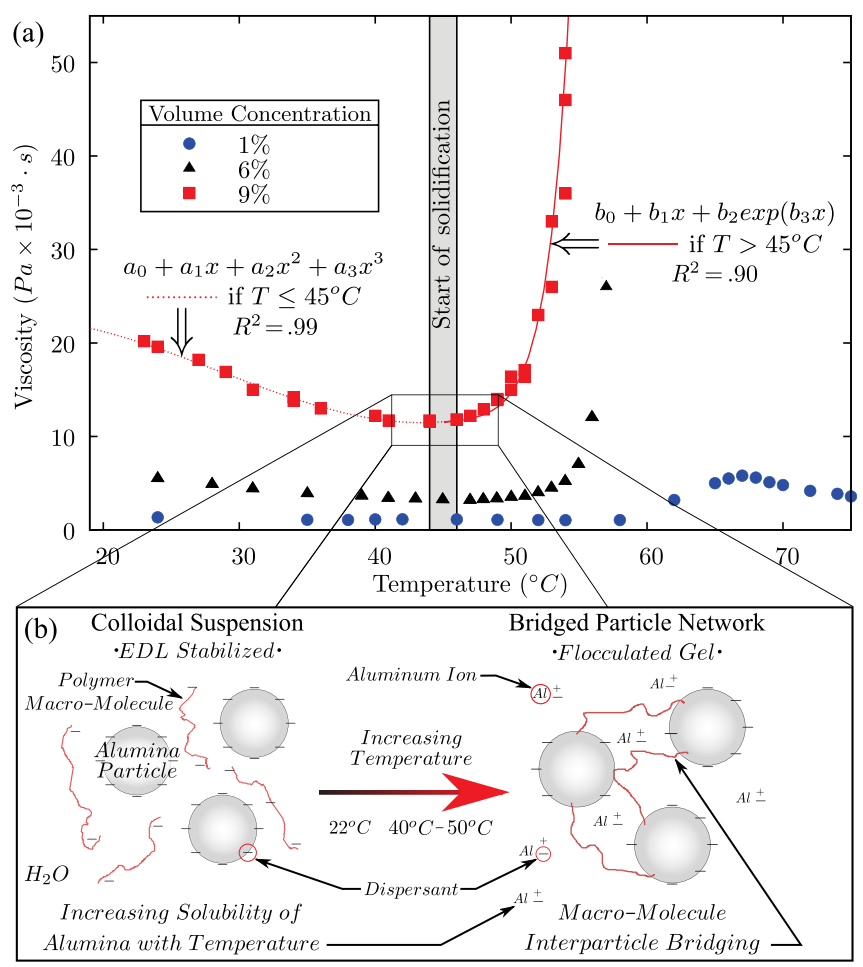

FIG. 1. (a) Viscosity measurements of $20 \mathrm{~nm}$ alumina in $\mathrm{H}_{2} \mathrm{O}$ at a shear rate of $20 \mathrm{~s}^{-1}$ for various volume concentrations show an increase in viscosity as a result of Temperature Induced Forming. (b) Illustration of the TIF process where interparticle bridges form at elevated temperatures as a result of ionizing alumina and weakening EDL forces. $a_{0}=13.81, a_{1}=1.45$, $a_{2}=-7.08 \times 10^{-2}, \quad a_{3}=8.31 \times 10^{-4}, \quad b_{0}=11.85, \quad b_{1}=-1.00 \times 10^{-2}$, $b_{2}=1.75 \times 10^{-13}, b_{3}=6.12 \times 10^{-1}$. TIF illustration is based on work by Sigmund et al. ${ }^{16}$

ratio $Q=q_{d} / q_{c}$, where $q_{d}$ and $q_{c}$ are the flow rates of the dispersed and continuous liquids, respectively, is another critical parameter for manipulating the size and frequency of confined droplets. ${ }^{7,10,18}$ In our experiments, the flow rates are controlled via separate programmable syringe pumps and remain constant once the desired drop size is achieved. In addition, for $C a<0.015$, changes to the viscosity ratio have little to no effect on the resulting droplet volumes and frequencies; however, for higher capillary numbers, droplet volumes and frequencies are strongly influenced by the viscosity ratio. ${ }^{10}$

For alumina suspensions, the viscosity can be adjusted either by changing concentration or by heating as illustrated in Fig. 1. If only the dispersed phase viscosity is changed, the viscosity ratio will also change, but both the capillary number and flow rate ratio remain unchanged if the continuous fluid properties and all dispensed flow rates remain constant. In this scenario, the droplet volume/frequency will undoubtedly shift due to the change in dispersed Reynolds number and viscous shear along the drop surface. Therefore, for a given capillary number and flow rate ratio, multiple drop sizes can exist for different concentrations of the same dispersed liquid suspension, making the capillary number/ flow rate ratio parameter combination on its own insufficient for characterizing drop sizes in this system. To capture the effects of changing the viscosity ratio on the resulting droplet volume, Fig. 2 shows droplet volume changes for three different types of liquid droplet emulsions in silicone oil such

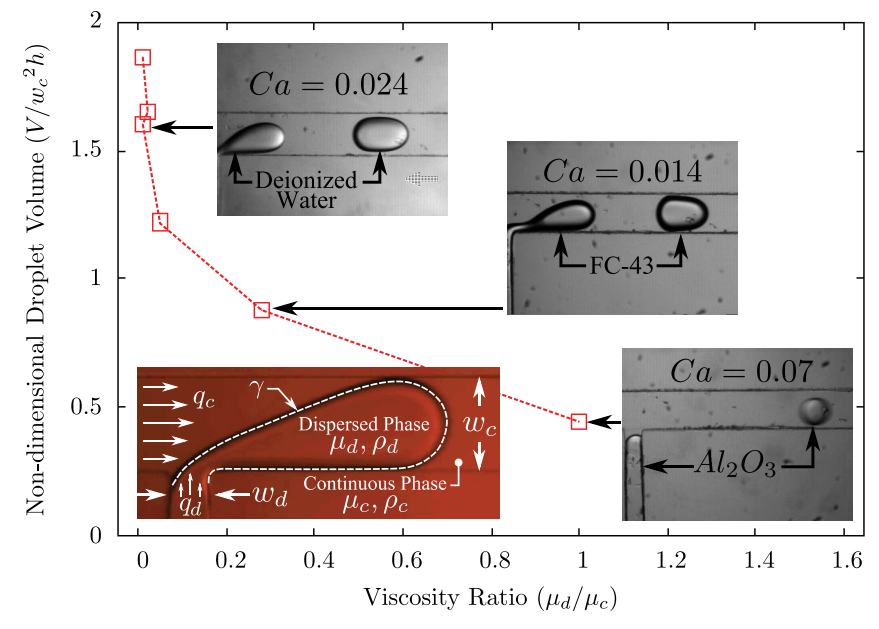

FIG. 2. Experimental results of non-dimensional droplet volume as a function of viscosity ratio and features of the T-junction used to describe key parameters used for experimentation.

that a wide range of viscosity ratios could be affected. If the viscosity ratio increases, i.e., if the dispersed phase viscosity is increased, there will be an impact on the size of the resulting solid particles. This effect becomes even more pronounced for higher capillary numbers as suggested by de Menech et al. ${ }^{19}$ Particle size can also be affected by the syringe pumps; however, this effect was found to be minimal. These pumps have been shown to cause a $\pm 5 \%-10 \%$ fluctuation in the flow rate due to low frequency cyclic variations in the continuous and dispersed phase flow rates. ${ }^{10}$

In order to design a suitable microfluidic device with the appropriate heat flux to produce microsolids, a 3-dimensional simulation study was conducted using COMSOL Multiphysics. The numerical model provides only an estimate for the temperature of the continuous fluid along the centerline of the microchannel in the absence of alumina droplets. Fig. 3 illustrates a 2-dimensional plot of the device temperature along a vertical cut-plane positioned at the centerline as well as the temperature of the continuous $20 \mathrm{cSt}$ of silicone oil along the centerline, and consequently, the location where an alumina droplet would begin to solidify. The temperature profile of the oil is given along with the curve fit for alumina viscosity vs. temperature to predict the location and the degree to which an alumina droplet would solidify as it progresses along the heated section of the device. The droplet present in the channel would occupy regions away from the centerline, close to the hot walls, and as a first order estimate, is expected to be at temperatures closer to that of the centerline, counteracting the increased heat capacity of the dispersed fluid. The alumina droplet that was omitted from the thermal model has a much higher thermal conductivity and heat capacity when compared to the continuous oil flow. ${ }^{20}$ Therefore, the model illustrated in Fig. 3 is idealized to provide the centerline temperature for the single phase continuous oil flow to predict the dispersed flow temperature.

As the alumina droplet progresses along the heated channel, the temperature of the droplet increases steadily as it enters the heater domain represented by vertical dashed lines (Fig. 3). In this region, viscosity increases with temperature beyond the solidification temperature and continues to 


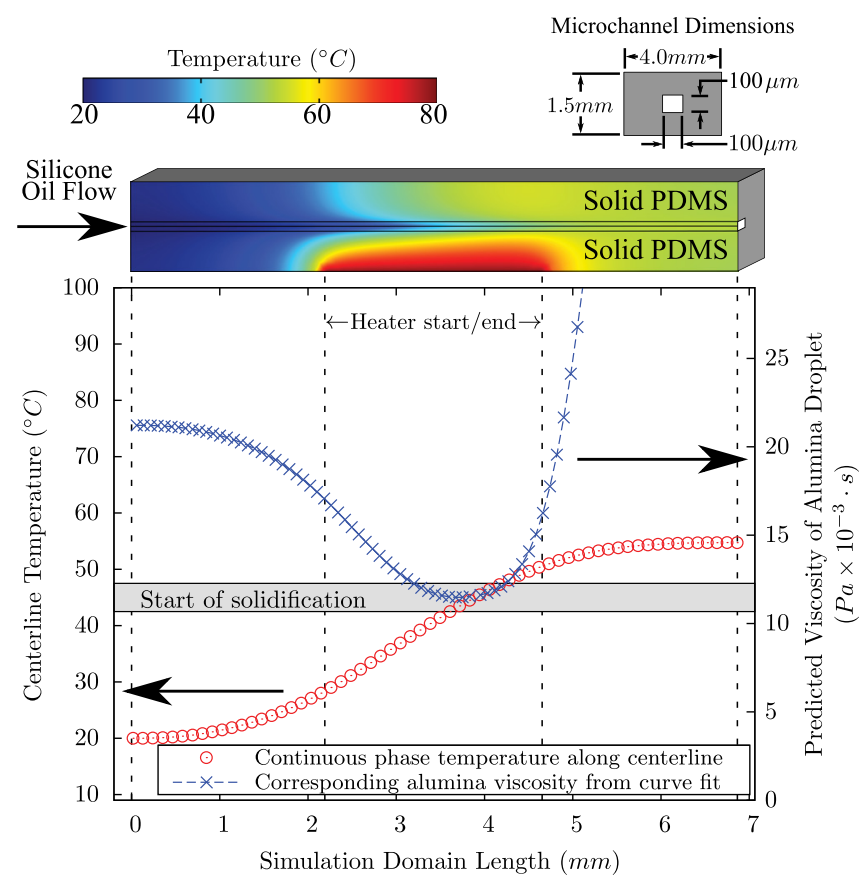

FIG. 3. Numerical temperature distribution of $20 \mathrm{cSt}$ of silicone oil along the centerline of microchannel without alumina droplet. Corresponding $9 \%$ volume concentration of alumina viscosity is superimposed utilizing the curve fit from Fig. 1 and the centerline temperature to indicate the solidification of alumina droplets along the microchannel domain. At $3.5 \mathrm{~mm}$ from the inlet, the oil approaches the solidification temperature for the alumina nanosuspension.

increases as the temperature is sufficient to induce solidification. The resulting solid formed by passage through the heated section is rigid enough to prevent coalescence and to preserve the morphology of the sphere. Solidification is initiated with the formation of a shell with a liquid core of decreasing solid concentration.

The mechanism of solidification relies on a suspension's ability to dissociate and form ions at elevated temperatures. Alumina, for example, sheds positively charged $\mathrm{Al}$ ions while in aqueous suspension as the temperature increases. This shedding of ions reduces the magnitude of the EDL repulsive forces and aids in the initiation of flocculation similar to other commonly used mechanisms for solidification. ${ }^{16}$ This method, however, differs from other methods in that the polymeric stabilizer in the suspension with negatively charged sites adheres to the newly developed surface sites on the alumina particle due to the dissociation of the $\mathrm{Al}$ ion. This progresses throughout the solution until irreversible flocculation occurs as illustrated in Fig. 1. ${ }^{16}$

This process suggests a transition from a stable nanoparticle suspension within the droplet resulting from EDL forces to an unstable suspension consisting of linked particleparticle networks. In this flocculated state, the ESD forces have been nullified by the ionizing of the alumina and the formation of inter particle bridges which bring the particles closer together. The proximity of the particles increases the Van der Walls attractive forces as suggested by the Derjaguin, Landau, Verwey, and Overbeek (DLVO) theory, and finally, the steric repulsive forces contributed by the surfactant are then overcome due to the ionization of the alumina particles and the formation of inter-particle bridging networks at the elevated temperatures. ${ }^{16,21}$
To illustrate the formation of a shell structure and the precipitation of water to the alumina-oil interface at temperatures much greater than the gelation temperature, $T_{g}$, a pendant droplet experiment is devised, where a droplet of $9 \%$ volume concentration of alumina is suspended in $95^{\circ} \mathrm{C}$ of silicone oil (Fig. 4). At $t=0 \mathrm{~s}$, the droplet is largely unaffected by the presence of the heated oil where its initial diameter, $D_{0}$ is $1.47 \mathrm{~mm}$ and $D / D_{0}=1$. At $t=1 \mathrm{~s}$, the formation of the outer shell occurs and can be determined by the transition of the translucent edges of the droplet to opaque where the normalized diameter remains at unity. At $t=4 \mathrm{~s}, D / D_{0}=.99$, and the initiation of precipitation can be detected where the water wets the steel needle used to suspend the droplet. Precipitation continues until about $t=132 \mathrm{~s}$, where the final normalized diameter is within $1 \%$ of the final diameter of $1.32 \mathrm{~mm}$.

The experiment illustrates a scenario in which two competing agglomeration mechanisms occur. The agglomeration of particles at the interface results in a tensile state of the free surface due to decreasing interparticle spacing. This occurs as the temperature of the droplet continues to increase toward the center beyond $T_{g}$. As the shell begins to increase in thickness, the normalized diameter continues to deviate from unity where the water, formerly dispersing the particles in suspension, acts as bystander in the agglomeration process. Temperature induced forming (TIF) reduces interparticle spacing. As a result, the tensile forces squeezees the water from the suspension and injects it into the oil-alumina interface forming satellite droplets of water wetting the new alumina shell. These water droplets wet the alumina solid as the alumina surface is hydrophillic when compared to the oil and as a result, wets with a relatively large contact angle. For a suspension of particles in a fluid, which is miscible with the heated surroundings near the dispersing fluids saturation temperature, the precipitation of water from the alumina shell would be accompanied not only by evaporation and mechanical squeezing due to TIF but also by diffusion across the interface. ${ }^{15}$ The presence of water formation at the oilalumina interface occurs mostly due to mechanical squeezing and evaporation. A similar phenomenon occurs in the microchannel, although the satellite droplets were not observed.

Consideration of the proposed oil water model for the agglomeration of alumina in water implies that the final droplet diameter depends not only on the initial diameter of the droplet formed at the T-junction but also on the volume concentration of solids in suspension. Given the same

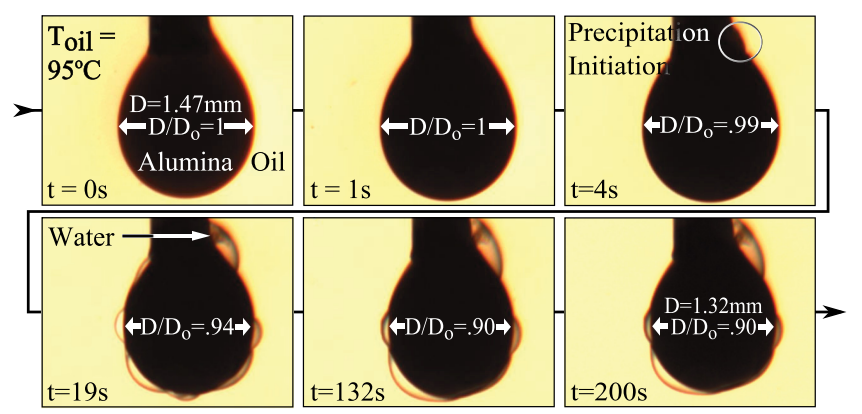

FIG. 4. Pendant droplet of $9 \%$ volume concentration of alumina heated in a bath of $95^{\circ} \mathrm{C}, 20 \mathrm{cSt}$ silicone oil at various times demonstrating the precipitation of water from the droplet as agglomeration at the interface occurs. 

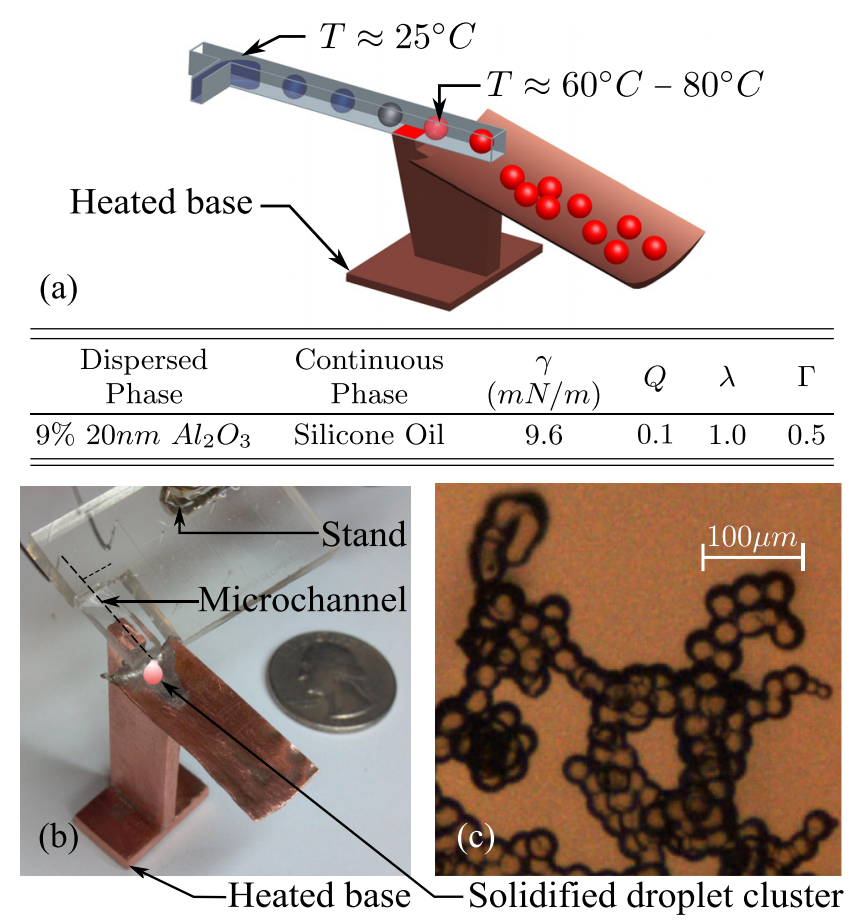

FIG. 5. (a) Heating of the alumina droplet. Droplet forms at room temperature and progresses through the heated microchannel until the droplet exits at $\approx 70^{\circ} \mathrm{C}$. Key parameters are tabulated, which describe the flow configuration utilized to produce solid microspheres. Flow rate ratio: $Q=q_{d} / q_{c}$, width ratio: $\Gamma=w_{d} / w_{c}$, viscosity ratio: $\lambda=\mu_{d} / \mu_{c}$, interfacial tension: $\gamma$. (b) Experimental setup used to heat the microchannel and the basis of the proposed numerical model Fig. 3. (c) Image of final solid droplets formed in the device. Average diameter of $30 \mu \mathrm{m} \pm 0.3 \mu \mathrm{m}$ with a standard deviation of $2.1 \mu \mathrm{m}$.

experiment with high solid content, one would expect the formation of a solid with minimal shrinkage due to the reduction of available water for precipitation and the already compact interparticle spacing of alumina particles.

Now equipped with a physical understanding of the solidification of alumina suspensions through TIF, we present a device to produce $30 \mu \mathrm{m}$ solid microspheres using a T-junction. This device was trimmed of excess material around the microchannel to reduce thermal resistance as solid polydimethylsiloxane (PDMS) possesses a relatively low thermal conductivity. The heat was delivered to the microchannel through the use of a copper pedestal. This structure was positioned on a heater and due to the high thermal conductivity, apparatus effectively carried heat into the microchannel through a stud at the height of the pedestal (see red heater patch in Fig. 5(a)). The temperature of the droplets begins to rise as they pass the heater. At this point, the temperature of the oil and the outer layer of the alumina reach $T_{g}$.

The microchannel was held in position by an adjustable stand, where the downward angle of the microchannel facilitated the formation of a pendant drop at the tip. The modifications made to the device allowed for the production of solid particles. Flow rates for the continuous and dispersed streams were selected $\left(q_{d}=100 \mu \mathrm{l} / \mathrm{h}\right.$ and $\left.q_{c}=1000 \mu \mathrm{l} / \mathrm{h}\right)$ for stable droplet production, and the heater was adjusted to produce temperatures at the tip of the pedestal of $\approx 80^{\circ} \mathrm{C}$. Other critical parameters governing droplet formation at a
T-junction for the production of solids in the microchannel are tabulated in Fig. 5(a). The configuration utilized to achieve solidification resulted from the modification of channels originally designed for the production and analysis of droplets. Channel networks used to route droplets to waste containers were removed to allow the droplets to flow uninhibited to an outlet for collection (Fig. 5(b)). Once steady state conditions were established and clump-free droplet formations emerged at the exit, the pendant droplet containing oil and alumina fell onto the trough structure on the copper pedestal and travelled to the tip for collection on a petri dish where observations could be made (Fig. 5(c)). The particles were imaged with a microscope along with a Ward's Science brand calibrated micron scale at the same magnification and focal distance as the particles. The particle diameters were interrogated using photo editing software and the resulting pixel measurements were converted to micrometers. The average droplet size of a sample population of 21 microspheres was $30 \mu \mathrm{m} \pm 0.3 \mu \mathrm{m}$ with a standard deviation of $2.1 \mu \mathrm{m}$.

In summary, a method for the production of $30 \mu \mathrm{m}$ alumina microspheres has been developed that preserves the nanoscale properties. This research was conducted with the understanding of the temperature sensitive rheological properties of the colloidal suspensions through temperature induced forming. Insight was also developed with "separate effects" experiments in T-junctions to produce the desired droplets using alumina suspensions and also in larger scale pendant droplets.

${ }^{1}$ T. Squires and S. Quake, Rev. Mod. Phys. 77, 977 (2005).

${ }^{2}$ H. Stone, A. Stroock, and A. Ajdari, Annu. Rev. Fluid Mech. 36, 381 (2004).

${ }^{3}$ J. Voldman, M. Gray, and M. Schmidt, Annu. Rev. Biomed. Eng. 1, 401 (1999).

${ }^{4}$ K. Jain, Pharmacogenomics 1, 289 (2000).

${ }^{5}$ D. Beebe, G. Mensing, and G. Walker, Annu. Rev. Biomed. Eng. 4, 261 (2002).

${ }^{6}$ P. Kenis, R. Ismagilov, and G. Whitesides, Science 285, 83 (1999).

${ }^{7}$ A. Gupta, S. Murshed, and R. Kumar, Appl. Phys. Lett. 94, 164107 (2009).

${ }^{8}$ A. Gupta and R. Kumar, Microfluid. Nanofluid. 8, 799 (2010).

${ }^{9}$ A. Gupta and R. Kumar, Phys. Fluids 22, 122001 (2010).

${ }^{10}$ J. D. Wehking, M. Gabany, L. Chew, and R. Kumar, "Effects of viscosity, interfacial tension, and flow geometry on droplet formation in a microfluidic T-junction," Microfluid. Nanofluid. (to be published).

${ }^{11}$ G. Christopher and S. Anna, J. Phys. D: Appl. Phys. 40, R319 (2007).

${ }^{12}$ S. Xu, Z. Nie, M. Seo, P. Lewis, E. Kumacheva, H. Stone, P. Garstecki, D. Weibel, I. Gitlin, and G. Whitesides, Angew. Chem. 117, 734 (2005).

${ }^{13}$ M. Seo, Z. Nie, S. Xu, M. Mok, P. Lewis, R. Graham, and E. Kumacheva, Langmuir 21, 11614 (2005).

${ }^{14}$ T. Nisisako and T. Hatsuzawa, Microfluid. Nanofluid. 9, 427 (2010).

${ }^{15}$ A. Fang, C. Gosse, C. Gaillard, X. Zhao, and J. Davy, Lab Chip 12, 4960 (2012).

${ }^{16}$ W. Sigmund, N. Bell, and L. Bergström, J. Am. Ceram. Soc. 83, 1557 (2000).

${ }^{17}$ J. D. Wehking, L. Chew, and R. Kumar, Appl. Phys. Lett. 103, 054101 (2013).

${ }^{18}$ P. Garstecki, M. J. Fuerstman, H. A. Stone, and G. M. Whitesides, Lab Chip 6, 437 (2006).

${ }^{19}$ M. De Menech, P. Garstecki, F. Jousse, and H. A. Stone, J. Fluid Mech. 595, 141 (2008).

${ }^{20}$ D. Milanova, R. Kumar, S. Kuchibhatla, and S. Seal, in Proceedings of 4 th International Conference on Nanochannels, Microchannels, and Minichannels, Limerick, Ireland (ASME, 2006).

${ }^{21}$ Y. Yang and W. Sigmund, J. Eur. Ceram. Soc. 23, 253 (2003). 